\title{
Emprego de pote fumígeno para proteção de insetário e sua ação residual contra triatomíneos, em condições de laboratório
}

\author{
The use of insecticide fumigant canister to protect insectarium and its residual \\ effect against triatomine bugs, in laboratory conditions
}

\author{
João Carlos Pinto Dias¹ e Eduardo N. Zerba²
}

\begin{abstract}
Resumo Pote fumígeno à base de piretroides e diclorvos foi empregado para desinsetizar um insetário de triatomíneos, contaminado com baratas e formigas. Retirados os triatomíneos durante a aplicação, o produto mostrou-se muito eficiente contra os insetos invasores, eliminando-os completamente em 48 horas. Monitorandose a ação triatomicida através de frascos sentinelas com adultos, ninfas e ovos de Triatoma infestans, Panstrongylus megistus e Rhodnius neglectus, não se observou ação ovicida, mas sobre os adultos e ninfas até pelo menos 72 horas após a fumigação, no recinto fechado.
\end{abstract}

Palavras-chaves: Doença de Chagas. Triatomíneos. Controle. Pote fumígeno.

Abstract An insecticide fumigant canister based on synthetic pyrethroids and dichlorvos was employed against cockroaches and ants which were invading an insectarium used for rearing triatominae. After removal of the Triatominae, the canister was activated and found to kill all the invading insects within 48 hours. Possible residual action against triatomines was then monitored by a 24-hour exposure of eggs, nymphs and adults of Triatoma infestans, Panstrongylus megistus and Rhodnius neglectus in the treated insectarium. No ovicidal action was observed but some mortality of adults and nymphs of the three species was observed up to 72 hours after the fumigation.

Key-words: Chagas' disease. Triatomines. Control. Fumigant canister.

Dentre os recursos na luta química contra insetos, nos últimos 70 anos uma série de moléculas têm sido empregadas tanto em Saúde Pública como em Agronomia, sendo mais conhecidos os inseticidas órgano-clorados, órgano-fosforados, carbamatos e piretróides. De acordo com o alvo e situação epidemiológica, formulações várias são utilizadas, como pó molhável, concentrados emulsionáveis e pinturas inseticidas para aplicação em paredes e ação por contato, aerossóis e micropartículas em ultrabaixo volume, larvicidas para uso aquático, etc ${ }^{11} 15$. Para o uso espacial, há vários anos têm sido usados vapores e fumaças de piretro, compostos clorados, órganofosforados (DDVP), carbamatos e piretróides sintéticos, pretendendo-se uma grande difusão em ambientes fechados e penetração otimizada em nichos e esconderijos de mui difícil acesso por outras formulações ${ }^{15}$. Foi o caso clássico do combate a baratas em galerias de esgotos sanitários com vapores de Vapona ${ }^{\circledR}$ (DDVP) e, a partir dos anos 80 , os trabalhos de controle de triatomíneos domiciliados com artefatos simples de produção de fumaça inseticida (potes e pastilhas fumígenos), particularmente desenvolvidos na

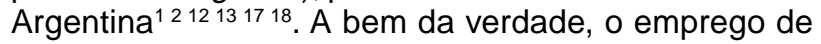
gases tóxicos contra os transmissores da doença de Chagas já fora testado por Emmanuel Dias, em 1944, aplicando cianogás em cafuas de Bambuí, Brasil, com sucesso relativo sobre ninfas e adultos mas sem ação ovicida e residual, além de ser extremamente complexa a operação idealizada ${ }^{4}$. Posteriormente, vapores de hexaclorociclohexano foram testados contra triatomíneos, em terreno, com ação comprovada mas sem nenhuma vantagem prática sobre o produto aplicado sobre as paredes na formulação de pó molhável $^{12}{ }^{13}$. Já tradicionais fumaças repelentes de insetos, largamente disseminadas no mercado domosanitário, à base de flor de piretro e pentaclorofenato de sódio, não se mostraram com ação triatomicida em adultos, ovos ou ninfas de Triatoma infestans e Panstrongylus megistus ${ }^{8}$. Atualmente, os potes fumígenos têm alcançado grande difusão na Argentina, para aplicação intradomiciliar contra triatomíneos, em esquemas de vigilância entomológica com participação comunitária, sendo que a última versão desta arma

1. Centro de Pesquisas René Rachou da Fundação Oswaldo Cruz, Belo Horizonte; MG, Brasil. 2. Centro de Investigaciones de Plagas e Insecticidas, Buenos Aires, Argentina.

Endereço para correspondência: Dr. João Carlos Pinto Dias. Centro de Pesquisas René Rachou/FIOCRUZ. Av. Augusto de Lima 1715, Barro Preto, 30190-002 Belo Horizonte, MG, Brasil

Tel: $55313295-3566$

e-mail:jcpdias@cpqrr.fiocruz.br

Recebido para publicação em: 16/04/2001 
inseticida contém basicamente o composto Betacipermetrina, formulado com protetores de decomposição

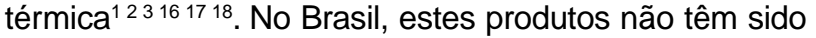
aplicados no programa governamental contra a doença de Chagas porque a imensa maioria dos focos atuais encontra-se no peridomicílio, onde sua ação é mínima ${ }^{910}$. Por outro lado, em vários centros de pesquisa sobre doença de Chagas, criações de triatomíneos são importantes e devem ser mantidas livres de outros insetos, que muitas vezes competem ou mesmo destroem os barbeiros, sendo necessário seu controle para que a criação se desenvolva eficientemente ${ }^{456}$. O presente artigo pretende informar, a partir de um caso concreto, sobre a utilização de potes fumígenos para a proteção de um insetário de triatomíneos contra outros insetos, aproveitando-se para medir-se uma eventual ação residual do produto contra os vetores da doença de Chagas, em ambiente fechado e sob condições de laboratório.

\section{MATERIAL E MÉTODOS}

Insetário principal do Laboratório de Triatomíneos e Epidemiologia da Doença de Chagas do Centro de Pesquisas René Rachou/FIOCRUZ, Belo Horizonte, Brasil, totalmente fechado, escuro e sem janelas, com área total de $24 \mathrm{~m}^{2}$ e volume total de $84 \mathrm{~m}^{3}$, albergando cerca de 64.000 triatomíneos de 11 espécies diferentes, para criação em frascos de vidro, assestados sobre prateleiras de madeira, segundo a técnica original de Dias $^{5}$. Semiclimatizado através de termostato, aquecedores e umidificador, o cômodo mantém-se em uma temperatura anual estável entre 27 e 29 graus Celsius e umidade de $70-80 \%$. Com paredes e teto de alvenaria rebocada e pintada a óleo, o piso era de ripas de madeira sobre azulejos, onde começaram a aparecer formigas (Monomorium sp) e baratas (Blatella germanica), provavelmente transportadas passivamente por meio de galinhas e pombos que semanalmente são trazidos de um biotério externo para a alimentação dos triatomíneos. Tendo sido tentado o controle com limpeza e aplicação de fenol e ácido bórico em iscas açucaradas, por três oportunidades, não houve resultado completo, o que motivou decisão por experimentar-se o pote fumígeno, trazido por um de nós da Argentina. Para tanto, foram transferidos todos os frascos de triatomíneos do insetário principal para outro cômodo, aplicando-se no insetário vazio 2 potes do produto Musal ${ }^{\circledR}$ de $155 \mathrm{~g}$ produzido pela firma então denominada Chemotécnica Syntial SA, de Buenos Aires, que tem a seguinte composição por $100 \mathrm{~g}$ : asimetrina (hoje denominada beta-cipermetrina) 1,3g, permetrina $2 \mathrm{~g}$, diclorvos $7 \mathrm{~g}$ e substâncias fumígenas, inertes e estabilizantes q.s.p. $100 \mathrm{~g}^{2}$. Este pote tem um pavio central que deve ser acendido manualmente e é previsto para liberar, em poucos minutos, vapores dos princípios ativos, em rápida seqüência, com o rendimento de 1 pote por $200 \mathrm{~m}^{3}$ de volume. Os primeiros vapores, neste caso, correspondem aos piretroides (asimetrina e permetrina), com forte efeito irritativo e de knock-down, seguindo-se o fosforado (diclorvos), admitindo-se um certo sinergismo dos compostos, nesta formulação ${ }^{217}$. Conforme indicado, o cômodo fumigado deve permanecer fechado por mais 120 minutos após a liberação total da fumaça inseticida, isto permitindo sua ampla difusão por todas as frestas e espaços disponíveis ${ }^{1318}$. O presente trabalho realizou-se nas condições padronizadas do insetário, mantido em plena obscuridade e completamente fechado. Neste trabalho, para controle do efeito residual do produto e segurança dos insetos da criação, entre o momento imediatamente após a aplicação do produto e 8 dias após foram levados ao recinto fumigado 9 frascos de triatomíneos que ali eram deixados por 24 horas, para observação de possível mortalidade durante mais dez dias, fora daquele recinto. Utilizaram-se 3 espécies de triatomíneos em cada exposição, sendo 1 frasco com adultos alimentados, 1 frasco com ninfas de $3^{\circ}$ estádio alimentadas e 1 frasco com ovos recém postos, para Panstongylus megistus, Triatoma infestans e Rhodnius neglectus. Os frascos para exposição e controle eram de vidro neutro cristalino, cilíndricos e com as dimensões de $12 \mathrm{~cm}$. de diâmetro por $12 \mathrm{~cm}$. de altura, cuja parte superior era vedada com uma gaze. No interior, uma sanfona de papel de filtro grosso servia de suporte aos triatomíneos, conforme a técnica de Dias $^{5}$. Em paralelo a cada frasco exposto ao recinto fumigado, um frasco identicamente montado era deixado como controle em recinto livre de fumigação, com leituras diárias nos mesmos momentos. Durante os dias de observação foram anotados ainda os encontros de baratas e formigas, vivas ou mortas, no recinto tratado pelo pote fumígeno.

\section{RESULTADOS}

De modo geral, a ação inseticida foi intensa e de pronta resposta sobre as baratas e formigas invasoras, que já ao segundo dia pós fumigação não mais foram detectadas vivas, assim permanecendo pelos 90 dias subseqüentes. Sobre os triatomíneos expostos, houve mortalidade significativa de adultos e ninfas nas primeiras 72 horas pós fumigação, sem nenhum efeito sobre os ovos, que desalagaram normalmente entre 2 e 4 semanas após a exposição (95\% de viabilidade das ninfas, sem diferença com os ovos controle). Do quarto ao $7^{\circ}$ dia após o tratamento ainda ocorreram algumas mortes nos triatomíneos expostos, praticamente não mais se registrando mortes a partir do $8^{\circ}$ dia (Tabela 1 ). Não houve diferença significativa entre as três espécies estudadas, em termos de mortalidade. Similarmente, também não houve diferenças significativas entre os estádios evolutivos neste experimento, embora se tenha notado uma tendência aparente a maior mortalidade 


\begin{tabular}{|c|c|c|c|c|c|c|c|c|c|}
\hline \multirow{2}{*}{$\begin{array}{l}\text { Espécie } \\
\text { Estádio e } \\
\text { exposição }\end{array}$} & \multicolumn{9}{|c|}{ Estádio e exposição ao ambiente em diferentes tempos relativamente ao acionamento do pote } \\
\hline & $\begin{array}{l}\text { antes de } \\
\text { acionar }\end{array}$ & $\begin{array}{c}0 \text { horas } \\
\text { (durante) }\end{array}$ & $\begin{array}{c}24 \text { horas } \\
\text { (após) }\end{array}$ & $\begin{array}{c}48 \text { horas } \\
\text { (após) }\end{array}$ & $\begin{array}{c}72 \text { horas } \\
\text { (após) }\end{array}$ & $\begin{array}{l}4 \text { dias } \\
\text { (após) }\end{array}$ & $\begin{array}{l}6 \text { dias } \\
\text { (após) }\end{array}$ & $\begin{array}{l}8 \text { dias } \\
\text { (após) }\end{array}$ & $\begin{array}{r}10 \text { dias } \\
\text { (após) }\end{array}$ \\
\hline $\mathrm{Pm}-\mathrm{ad} / \mathrm{e}$ & 0,0 & 100,0 & 100,0 & 90,0 & 60,0 & 20,0 & 10,0 & 0,0 & 0,0 \\
\hline Pm-nf/e & 10,0 & 100,0 & 100,0 & 100,0 & 80,0 & 40,0 & 20,0 & 10,0 & 0,0 \\
\hline $\mathrm{Pm}-\mathrm{ad} / \mathrm{c}$ & 0,0 & 0,0 & 0,0 & 0,0 & 0,0 & 10,0 & 0,0 & 0,0 & 0,0 \\
\hline Pm-nf/c & 0,0 & 0,0 & 0,0 & 10,0 & 0,0 & 0,0 & 0,0 & 0,0 & 0,0 \\
\hline Ti-ad/e & 0,0 & 100,0 & 90,0 & 80,0 & 70,0 & 30,0 & 10,0 & 0,0 & 0,0 \\
\hline Ti-nf/e & 0,0 & 100,0 & 100,0 & 90,0 & 80,0 & 30,0 & 10,0 & 10,0 & 0,0 \\
\hline Ti-ad/c & 0,0 & 0,0 & 0,0 & 0,0 & 0,0 & 0,0 & 10,0 & 0,0 & 0,0 \\
\hline Ti-nf/c & 0,0 & 0,0 & 0,0 & 0,0 & 0,0 & 10,0 & 0,0 & 0,0 & 0,0 \\
\hline $\mathrm{Rn}-\mathrm{ad} / \mathrm{e}$ & 0,0 & 100,0 & 100,0 & 100,0 & 60,0 & 20,0 & 10,0 & 0,0 & 0,0 \\
\hline Rn-nf/e & 0,0 & 100,0 & 100,0 & 100,0 & 90,0 & 30,0 & 10,0 & 10,0 & 0,0 \\
\hline $\mathrm{Rn}-\mathrm{ad} / \mathrm{c}$ & 0,0 & 0,0 & 10,0 & 0,0 & 0,0 & 0,0 & 0,0 & 0,0 & 0,0 \\
\hline $\mathrm{Rn}-\mathrm{nf} / \mathrm{c}$ & 10,0 & 0,0 & 0,0 & 0,0 & 0,0 & 0,0 & 0,0 & 0,0 & 10,0 \\
\hline
\end{tabular}

Observações: 10 insetos por frasco de exposição. $P m=P$. megistus. $T i=$ T. infestans. $R n=R$. neglectus. ad/e = adulto exposto. ad/c = adulto controle. $\mathrm{nf} / \mathrm{e}=$ ninfa exposta. $\mathrm{nf} / \mathrm{c}=$ ninfa controle.

entre as ninfas. Observou-se que as mortes ocorridas após $04^{\circ}$ dia da fumigação, além de o serem em proporção significativamente menor que as dos primeiros dias, ocorriam em prazos mais lentos, tardando até 10 dias para todos os insetos morrerem.
A Tabela 1 explicita os dados de mortalidade para os adultos e ninfas de triatomíneos das três espécies e seus controles expostos ao ambiente antes do acionamento do pote, durante o mesmo e em diferentes dias de exposição a seguir, até 10 dias depois.

\section{DISCUSSÃO}

Este pequeno trabalho teve por primeira finalidade simplesmente tratar com potes fumígenos um insetário de triatomíneos contra a invasão de formigas e baratas, invasão esta que não é rara nas criações de barbeiros ${ }^{4}$. Mesmo que se busque manter estrita higiene do ambiente, a desinsetização química às vezes pode fazer-se necessária, mormente quando ocorrem duas situações especiais: a) quando a alimentação dos triatomíneos se faz a partir de sucção em animais vivos (geralmente aves) e é realizada dentro do próprio insetário, resultando em contaminação ambiental com fezes e urina da fonte alimentar, que também pode passivamente carrear predadores; $b$ ) quando o ambiente físico dispõe de possibilidades de abrigo a outros insetos como, no caso presente, piso de tábuas de madeira. Aqui existiam ambas as condições e a fumigação com o pote fumígeno foi uma alternativa extremamente eficiente para o controle dos insetos indesejáveis. Uma boa ação destes produtos sobre baratas (Periplaneta já fora descrita por Zerba et al, verificando-se $100 \%$ de mortalidade após exposição de 2 horas à fumaça inseticida ${ }^{17}$. Sobre formigas, embora não tivéssemos encontrado referências explícitas quanto ao uso dos potes, os princípios ativos aqui testados têm sido referidos como altamente eficientes contra várias espécies em outras formulações, como pó molhável e concentrados emulsionáveis ${ }^{11}$. Como complemento, informa-se aqui que posteriormente, o insetário estudado foi remodelado, trocando-se o piso por ladrilhos de alvenaria e instalando-se uma sala própria de alimentação fora do recinto de criação, com isto prevenindo-se novas infestações. Como objetivo secundário do trabalho, o emprego do pote foi monitorizado pela exposição programada de triatomíneos ao ambiente tratado, em dias sucessivos, para estabelecer-se com segurança o momento adequado de retorno dos insetos ao insetário. Isto ocorreu com segurança a partir do final da primeira semana, retornando-se os barbeiros no $10^{\circ}$ dia sem problema algum. Como se esperava, não houve ação do produto sobre os ovos expostos ao ambiente fumigado ${ }^{713}$ 17. É importante assinalar que em todas as exposições empregaram-se ovos jovens, ou seja, com menos de 7 dias após a oviposição. Como se sabe, proximamente ao desalagamento ocorre início de abertura do opérculo, o que proporciona a morte de algumas larvas antes da eclosão quando há inseticidas no córion e/ou seus vapores no ambiente ${ }^{7}$. Já sobre as ninfas e adultos de três espécies, a ação do produto empregado mostrou-se claramente ativa durante a fumigação, como seria de esperar-se, aliás conforme todos os antecedentes e indicações desta formulação ${ }^{17}$. Isto está de acordo com as observações de terreno, realizadas principalmente com $T$. infestans e $T$. dimidiata, mostrando boa ação imediata destes potes em recintos 
fechados ${ }^{1518}$. No presente experimento observou-se uma clara ação residual do produto nos dois primeiros dias pós-fumigação, o que pode em parte explicar-se pelo fato do ambiente em pauta ser rigorosamente vedado, certamente retendo vapores e resíduos do inseticida ${ }^{71} 1417$. Em estudos pioneiros, Zerba et al não conseguiram demonstrar a presença de resíduos de DDVP e deltametrina em superfícies do piso, paredes e teto de ambientes fumigados com pote semelhante em recinto fechado e posteriormente arejado, aos 5 minutos e aos 15 dias após aplicação, apenas detectando resíduos superficiais de lindane aos 5 minutos ${ }^{17}$. Tudo indica que a principal ação de tais produtos se faz via aérea por inalação dos insetos, através de seus espiráculos abdominais, como observado por aqueles autores analisando o processo de abertura e fechamento do $4^{\circ}$ espiráculo de triatomíneos após a exposição ${ }^{17}$. As presentes observações ratificam esta assertiva, pois os triatomíneos expostos ao ambiente não tocavam as suas superfícies do recinto fumigado, desde que confinados em pequenos frascos de vidro, apenas respirando o ar ambiental. No presente estudo não se permitiu ventilação do ambiente e mortalidade observada nos triatomíneos expostos ainda prosseguiu, pelo menos até $04^{\circ}$ dia pós fumigação, mas já decrescendo significativamente a partir das primeiras $48-72$ horas (Tabela 1). Provavelmente, dois fatores principais foram envolvidos com o pequeno efeito residual detectado nesta observação: a) o ambiente fechado do laboratório (muito mais cerrado que o interior de casas rústicas, que inclusive são abertas algum tempo após a fumigação, para serem ventiladas e poderem entrar os moradores); b) o fato de os insetos do experimento estarem impedidos, em seus frascos, de escaparem de um ambiente hostil , portanto obrigados a respirarem o ar muito pouco renovado do insetário. Por outro lado, também os triatomíneos criados em laboratório durante muitas gerações sucessivas, soem ser mais frágeis que os triatomíneos de campo em várias situações de privação e hostilidade ${ }^{5}$. Como conclusão principal desta nota, assinala-se a praticidade e a eficácia do pote fumígeno empregado contra baratas e formigas invasoras de insetários de triatomíneos, indicando-se um período mínimo de 7 dias após a fumigação para retornar os barbeiros ao local fumigado. Reitera-se a boa ação imediata da fumaça inseticida sobre ninfas e adultos de triatomíneos (três espécies estudadas), observando-se ainda uma evidente ação residual do produto até pelo menos 72 horas pós-fumigação, em ambiente fechado. Não houve ação contra os ovos dos triatomíneos, quando expostos ao ambiente fumigado ainda longe de seu momento de eclosão.

\section{REFERÊNCIAS BIBLIOGRÁFICAS}

1. Audino PG, Susana de Licastro $S$ \& Zerba EN. Thermal behaviour and biological activity of pyrethroids in smoke-generating formulations. Pesticide. Science 55:1187-1193, 1999.

2. Centro de Investigaciones de Plagas e Insecticidas (CIPEIN) Informações Técnicas sobre o pote fumígeno CIPEIN-PF-7. Mimeo, Buenos Aires, Argentina, 2001.

3. Dias E. Xenodiagnóstico e algumas verificações epidemiológicas na moléstia de Chagas. Anales de la Novena Reunión de la Sociedad Argentina de Patología Regional 1: 89-119, 1935.

4. Dias E. Criação de triatomíneos em laboratório. Memórias do Instituto Oswaldo Cruz 33: 407-412, 1938.

5. Dias E. Um ensaio de profilaxia da moléstia de Chagas. Imprensa Nacional, Rio de Janeiro, 1945.

6. Dias JCP. Suscetibilidade de ovos e larvas de triatomíneos à ação do BHC. Revista Brasileira de Malariologia e Doenças Tropicais 17: 37-47, 1965.

7. Dias JCP. Chagas disease control in Brazil: which strategy after the attack phase? Annales de la Societé Bélgique de Medecine Tropicale 71 (supl 1): 75-86, 1991.

8. Dias JCP. Vigilância epidemiológica contra a doença de Chagas. Revista da Sociedade Brasileira de Medicina Tropical 26 (supl III): 39-44, 1993.

9. Dias JCP, Silva JC. Sobre alguns aspectos da profilaxia defensiva em doença de Chagas. Revista do Instituto de Medicina Tropical de São Paulo 11: 236-244, 1969.

10. Harwood RF, James MT. Entomology in Human and Animal Health. Seventh Edition. New York, Macmillan Publishing Co, 1979.

11. Organización Panamericana de la Salud. Iniciativa del Cono Sur (1991-1992): estado actual de las acciones para la eliminación de la transmisión vectorial e interrupción de la transmisión transfusional del Trypanosoma cruzi. Washington, doc. PNSP/ 92-18, 1992.

12. Perlowagora-Szumlewics A. A ação tóxica dos vapores do Hexaclorociclohexana sobre o Triatoma infestans. Revista Brasileira de Malariologia e Doenças Tropicais 5: 172-181, 1953.

13. Romaña C, Abalos J. Acción de los generadores de humo a base de "Gamexane" sobre los triatomideos y otros artrópodos. Nota previa. Sociedade de Biología de Tucumán (Argentina), Mimeo, 1947.

14. Secretaria de Salud de la Nación. Manual para la vigilancia de la transmisión de Chagas. Instituto Mario Fatala Chaben, Buenos Aires, Argentina, 1986.

15. World Health Organization. Chemical methods for the control of arthropod vectors and pests of public health importance. Geneva, 1984.

16. World Health Organization. Control of Chagas Disease. Report of a WHO Expert Committee. Geneva. WHO Technical Report Series 811, 1991.

17. Zerba E, Villar MIP, Casabé N, Wallace G. Physiological and toxicological basis of a fumigant canister with triatomicidal activity. Annals of the TDR Meeting on Social, Economic and Epidemiologicas Aspects of New Tools for Chagas' Disease Control. Geneva/Buenos Aires, p.32-41, 1986.

18. Zerba E, Wood E, Melgar F, Balzán D, De Licastro SA, Villar MIP, De Malkenson NC, Fontán A, Segovia S, De Arguello NV, Madohery L, Chary L, Lopez G. Ensayo de campo para la evaluación del pote fumígeno CIPEIN-PF3 en el control del Triatoma infestans. Chagas 5: 19-28, 1989. 